\title{
Money makes the World go round oder ohne Moos nix los
}

Es gibt viele Sprüche und Gedanken rund um das Geld. Geld ist für uns alle ein wichtiger Faktor. Im djb wird der Hauptteil der Arbeit ehrenamtlich geleistet. Mit den Mitgliedsbeiträgen begleichen wir die laufenden Kosten, wie z.B. für die Geschäftsstelle einschließlich Personalkosten, Fahrtkosten für die Kommissions-, Bundesvorstands- und Regionalgruppenbeiratssitzungen, einen großen Teil der Veranstaltungskosten und die Zuwendungen an die Landesverbände und Regionalgruppen.

\section{Spenden für unsere Projekte}

In unserem Newsletter vom 15. Dezember 2008 baten wir Sie um Spenden für unsere Projekte. Dank Ihrer Spenden sind wir der Realisierung unserer Vorhaben näher gekommen, haben jedoch noch nicht die ausreichenden finanziellen Mittel für die Durchführung von zwei jetzt anstehenden Projekten und bitten alle Mitglieder um eine Spende insbesondere für

- die Ausstellung und Buch „Juristinnen in der DDR“

- die Marie Munk-Gedenktafel

Bedenken Sie bitte: Auch die kleinste Summe hilft.

Teilen Sie auf Ihrer Überweisung bitte mit, für welches Projekt Sie spenden möchten. Bei Mehreinnahmen wird Ihre Spende für andere wichtige djb-Projekte verwendet. Weitere Projekte finden Sie auf unserer Homepage im Internet www.djb.de/ Spenden/Projekte.

Da wir eine Spendenliste in der djbZ und/oder auf unserer Webseite veröffentlichen möchten, bitten wir um Mitteilung, ob Sie als Spenderin namentlich genannt werden wollen.

\section{Absetzbarkeit der Spenden}

Der djb ist wegen Förderung der Wissenschaft nach dem letzten uns zugegangenen Freistellungsbescheid des Finanzamtes für Körperschaften I in Berlin vom 2.10.2007 für die Jahre 2003 bis 2006 von der Körperschaftsteuer und der Gewerbesteuer befreit. Spenden an den djb sind daher bis zu einer Höhe von 20 Prozent des Gesamtbetrags der Einkünfte (oder bei Unternehmen: bis zu vier Promille der Summe der gesamten Umsätze und der im Kalenderjahr aufgewendeten Löhne und Gehälter) steuerlich absetzbar. Als Nachweis beim Finanzamt genügt der Bareinzahlungsbeleg oder die Buchungsbestätigung bei einem Kreditinstitut, wenn die Spende 200,00 Euro nicht übersteigt. Bei Beträgen über 200,00 Euro stellt Ihnen die Geschäftsstelle automatisch eine Zuwendungsbestätigung aus. Auf Wunsch erhalten Sie selbstverständlich auch für geringere Beträge eine Zuwendungsbestätigung.

\section{Spenden auch online}

Seit kurzem ist auf der djb-Homepage auch die Möglichkeit für einmalige oder regelmäßige online-Spenden eingerichtet.

\section{Bet Debora e.V.}

Frauenperspektiven im Judentum

Driesener Straße 1 A, 10439 Berlin

BET DEBORA

Telefon. +49-30-44 18102

E-Mail: bet.debora@gmail.com

Deutscher Juristinnenbund e.V.

Bundesgeschäftsstelle

Anklamer Str. 38, 10115 Berlin

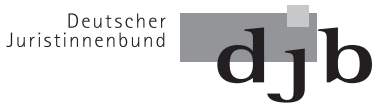

Telefon: +49-30-44 32 70-0, Telefax: +49-30-44 32 70-22

E-Mail: geschaeftsstelle@djb.de

\section{Wir bitten um Spenden für eine Gedenktafel zu Ehren von Dr. Marie Munk}

Bet Debora, eine 1998 ins Leben gerufene Initiative jüdischer Feministinnen, und der Deutsche Juristinnenbund (djb) möchten Dr. Marie Munk mit einer Gedenktafel ehren - an den Orten in Berlin, wo sie geboren wurde, aufgewachsen ist und ihre Kanzlei betrieb.

Marie Munk (geb. 4. Juli 1885 in Berlin, gest. 17. Januar 1978 in Cambridge/MA) gründete 1914 in Berlin zusammen mit Dr. Margarete Berent und Dr. Margarete Mühsam-Edelheim den „Deutschen Juristinnen-Verein“. Zu diesem Zeitpunkt waren Frauen noch nicht zur zweiten juristischen Staatsprüfung zugelassen. Der Juristinnen-Verein verfolgte den Zweck, die Interessen, insbesondere die beruflich-wissenschaftliche Fortbildung der Juristinnen zu fördern. 1933 löste der Verein sich auf, um der „Gleichschaltung" durch die Nationalsozialisten zu entgehen. Die Gründerinnen des Juristinnen-Vereins gingen ins Exil und kehrten - wie die meisten anderen jüdischen und als Jüdinnen verfolgten Juristinnen - nicht aus der Emigration zurück. 1948 wurde in Dortmund die Vereinigung „Weibliche Juristen und Volkswirte e.V." gegründet, heute „Deutscher Juristinnenbund e.V. (djb)“, die sich als Nachfolgerin des früheren Juristinnen-Vereins betrachtet. Weitere Informationen finden Sie bei www.djb.de.

Für die Kosten der Gedenktafel in Höhe von 1.500 Euro bitten wir um Spenden:

Bankverbindung Bet Debora e.V.:

Bank für Sozialwirtschaft, BLZ 100205 00, Kontonr. 3278700 IBAN: DE84 1002050000032787 00, BIC (SWIFT): BSFWDE33BER

Bankverbindung Deutscher Juristinnenbund e.V.:

Deutsche Bank Köln, BLZ 370700 24, Kto-Nr. 507315000

IBAN DE46 3707002405073150 oo, BIC (SWIFT) DEUT DE DBKOE

Spenden an Bet Debora oder an den djb sind steuerlich absetzbar. Sollten Sie eine Zuwendungsbestätigung für das Finanzamt benötigen, teilen Sie uns bitte Ihre Anschrift mit. 


\section{Juristinnen in der DDR}

Anlässlich der Herausgabe des „Juristinnenlexikons“ im Jahre 2005 und der damaligen Recherchen stellten wir fest, dass über DDR-Juristinnen - im Gegensatz zu DDR-Juristen - wenig bekannt ist. Einzige Ausnahme: Hilde Benjamin, die offenbar potentiellen Jurastudentinnen regelmäßig zur Abschreckung vorgeführt wurde. Zitat: „Willst du werden wie Hilde Benjamin?“ Das soll sich mit der Ausstellung ändern.

Das Projekt will darüber hinaus - insbesondere in einer wissenschaftlichen Begleitpublikation - die Frage beantworten, ob die ostdeutsche Justiz tatsächlich „Schauplatz einer weiblichen Erfolgsgeschichte“ war, weil die DDR einen weltweit einmalig hohen Anteil von Frauen unter den Juristen hatte. Denn dieser Anteil hing auch in der DDR in den verschiedenen juristischen Berufsgruppen stark mit deren Prestige zusammen. In der sozial höher angesiedelten Anwaltschaft waren Frauen nur in geringer Zahl vertreten. Von den Notaren jedoch, die in der DDR einen eher niedrigen sozialen Status innehatten, waren zum Zeitpunkt der deutschen Einheit 67,8 Prozent weiblich. Auch der Anteil von Frauen in der Justiz wurde kleiner, je höher die Position in der Justizhierarchie war. 1979 stellten sie zwar 51,1 Prozent der Richter an den Kreisgerichten, aber nur 23,2 Prozent der Direktoren. Auch in der DDR griffen damit offenbar alte Mecha- nismen, die zu einem deutlichen Ungleichgewicht zwischen den Geschlechtern führten.

Eine Wanderausstellung soll anhand von Tafeln mit 12 Einzelbiografien von Juristinnen

- aus verschiedenen „Generationen“: 1. vor Ende des 2. Weltkriegs ausgebildete Juristinnen, 2. in der DDR ausgebildete Juristinnen, die auch ausschließlich in der DDR berufstätig waren (dazu gehören insbes. die Volksrichterinnen), 3. in der DDR ausgebildete Juristinnen mit geteilter Berufsbiografie und

- Berufsgruppen: Richterin, Rechtsanwältin, Notarin, Staatsanwältin, Wissenschaftlerin, Verwaltungsjuristin (auch Polizei, Armee, Repressionsapparat), Justiziarin

einen Einblick in die Ausgestaltung der juristischen Berufe in der DDR aus der Genderperspektive ermöglichen. Die Biografien werden durch allgemeine Tafeln mit Hintergrundinformationen zur Situation der DDR-Justiz und der Frauen in der DDRJustiz sowie im Berufsleben ergänzt.

Die rechtshistorische Forschung soll in einer begleitenden Publikation vertieft und auch anhand von Statistiken, Fallstudien usw. sehr viel detaillierter werden, als dies auf den Ausstellungstafeln möglich ist.
Wenn Sie diese Möglichkeit nutzen möchten, so gelangen Sie unter www.djb.de/Spenden dorthin.

\section{Sponsoring durch Kanzleien oder Unternehmen}

Der Bundesvorstand hat im Jahre 2008 beschlossen, auch moderne Arten der Finanzierung wie das Sponsoring zu nutzen. Beim Sponsoring werden Finanzmittel, Sachleistungen und Dienstleistungen seitens des Sponsoring-Gebers (in der Regel ein Wirtschaftsunternehmen) gegen Image- und Know-howTransfer, Kommunikationsleistungen, Logoplatzierungen, Emotionalisierungen sowie verkaufsfördernde Maßnahmen seitens des Sponsoring-Nehmers ausgetauscht. Dieser Austausch basiert regelmäßig auf einem klaren Vertrag.
Sollten Sie in Ihrer Kanzlei oder in Ihrem Unternehmen für die Vergabe von Sponsoring-Geldern verantwortlich sein, würden wir uns freuen, wenn Sie mit uns Kontakt aufnehmen. Sponsoringbeauftragte ist Vizepräsidentin Renate Maltry (E-Mail: renate.maltry@djb.de).(Renate Maltry, Dagmar Brinkmann)

Für den djb danke ich den bisherigen Spenderinnen ganz herzlich! Insbesondere der Realisierung unseres Gedenktafel-Projekts sind wir einen großen Schritt näher gekommen.

Jutta Wagner

Präsidentin

\section{Ausschreibung}

\section{Arbeitsstab „Berufsorientierung, Karriereplanung, Mentoring“ \\ - Vorsitz und Mitgliedschaft -}

Der Arbeitsstab „Berufsorientierung, Karriereplanung, Mentoring “ sucht zur Betreuung vorhandener und der Entwicklung neuer Projekte eine Vorsitzende und weitere Mitglieder.

Informationen über die bisherige Arbeit sind auf der djbWebseite abrufbar: http://www.djb.de/karriere/arbeitsstab-berufsorientierung-karriereplanung-mentoring/
Kolleginnen, die mitarbeiten möchten, werden gebeten, sich für den Vorsitz und/oder die Mitgliedschaft schriftlich bis zum 15. April 2009 über die Geschäftsstelle beim Bundesvorstand zu bewerben. Die Bewerbung sollte in kurzer Form Angaben darüber enthalten, welche Projekte Sie interessieren und vielleicht auch selbst vorschlagen möchten. 